\title{
Thermoluminescence energy response of germanium doped optical fibre using Monte Carlo N-particle code simulation
}

\begin{abstract}
Over the last decade, the investigation of thermoluminescence (TL) dosimetry has expanded enormously. Many phosphor materials have been studied, become commercially available and have been applied to many areas of ionizing radiation dosimetry. This study is focusing on energy response of commercially available silicon dioxide optical fibre subjected to photon irradiation. The result obtained will be compared with energy response of TLD 100. The TL response of various photon energy, ranging from $20 \mathrm{keV}$ to $6 \mathrm{MeV}$, was investigated as energy absorbed in the TL material. The study was carried out using Monte Carlo NParticle transport code version 5 (MCNP5). The inputs parameters include in this study are geometry specification, source information, material information and tallies. Tally F6 is use in this simulation. Even though the same pattern of response can be seen from each material, the simulation shows that the fibre optic had a greater response than TLD 100 in the lower energy ranges but slightly lower response than TLD 100 in the higher energy ranges.
\end{abstract}

Keyword: Energy response; Ge-doped optical fibre; MCNP5; Thermoluminescence 